\title{
Using Fuzzy Set as a Controller on the Lateral Dynamic of Autopilot to Reducing the Effect of the Atmospheric Disturbance
}

\author{
Muna Hadi Saleh, Noor Abdul Razzaq Raheem \\ Assist. Prof. Dr, Muna Hadi Saleh, Department of Electrical Engineering ,College of Engineering \\ drmuna4@yahoo.com \\ Noor Abdul Razzaq Raheem, Department of Electrical Engineering, College of Engineering \\ noorahmed201054@yahoo.com
}

\begin{abstract}
In this paper, used fuzzy set as a controller to control roll and yaw angles in lateral dynamic of the autopilot system for jet transport using MATLAB/SIMULINK. Autopilot systems are complex devices that demand exact control and stability. In this paper explained the lateral dynamic on automatic pilot and studied the effect of atmospheric disturbances on the stability then designed the fuzzy set controller to reduce three type of disturbance which faced the pilot during the flight.
\end{abstract}

\section{Keywords}

Lateral Autopilot, Roll angle, Yaw angle, Fuzzy set.

\section{Council for Innovative Research}

Peer Review Research Publishing System

Journal: INTERNATIONAL JOURNAL OF COMPUTERS \& TECHNOLOGY

Vol. 15, No. 2

www.ijctonline.com, editorijctonline@gmail.com 


\section{INTRODUCTION}

The autopilot is a system planned to lead a vehicle without an assist. Due to increase in flight hours and complexity of modern day flight it become necessary to provide aircraft with autopilot. Thus dependability and uniformity of an autopilot system become a pivotal role in a flight. The increased complexity and request to premium accuracy has made the procedure of evaluating the autopilot for consistency a difficult process. They involve complex control systems [1].

In recent years, several extensions of knowledge discovery methods have been developed on the basis of Fuzzy Set Theory (FST). Many patterns of interest are inherently vague; fuzzy approaches allow for modeling them in a more adequate way and thus enable the discovery of patterns that would otherwise remain hidden. Related to this, fuzzy approaches are often more robust toward a certain amount of variability or noise in the data, a point of critical importance in many practical application fields [2].

In this paper, In the first section, lateral dynamic modeling of the jet transport aircraft will be delivered. In the second section we explain the force of atmospheric disturbances on the constancy of the aircraft. And fuzzy set will be designed as a controller in autopilot system in the third section. Then discussed the results of this configuration.

\section{2-LATERAL AUTOPILOT MODE}

There are three axes of motions lateral, longitudinal and vertical axes. The lateral axis passes through the plane from wingtip to wingtip. Rotation around this axis is the pitch. Pitch changes the vertical guidance that shows the nose of the plane. The elevators are the primary control surfaces for pitch, the longitudinal axis, badges through the plane of the nose to rise. Revolution about this axis is called roll. Rolling motion to change the direction of the aircraft wings with respect to the landing of the force of gravity [3].

The lateral mode in autopilot was designed primarily to keep the wings level and hold the aircraft on a desired heading. The lateral mode consists of two inputs the aileron input and rudder input as shown in Figure (1). A vertical gyro was used for the purpose of keeping the wings level, and a directional gyro was used for the heading reference. Lateral and directional modes involve rolling motions and yawing motions. Motions in one of these axes almost always couple into the other so the modes are generally discussed as the Lateral Directional Modes.

There are three types of possible lateral-directional dynamic motion: roll subsidence mode, spiral mode, and Dutch roll mode. Dutch roll and cause observed in the yaw rate and sideslip response from the aileron deflection by moment deviation caused by the aileron deflection [4].

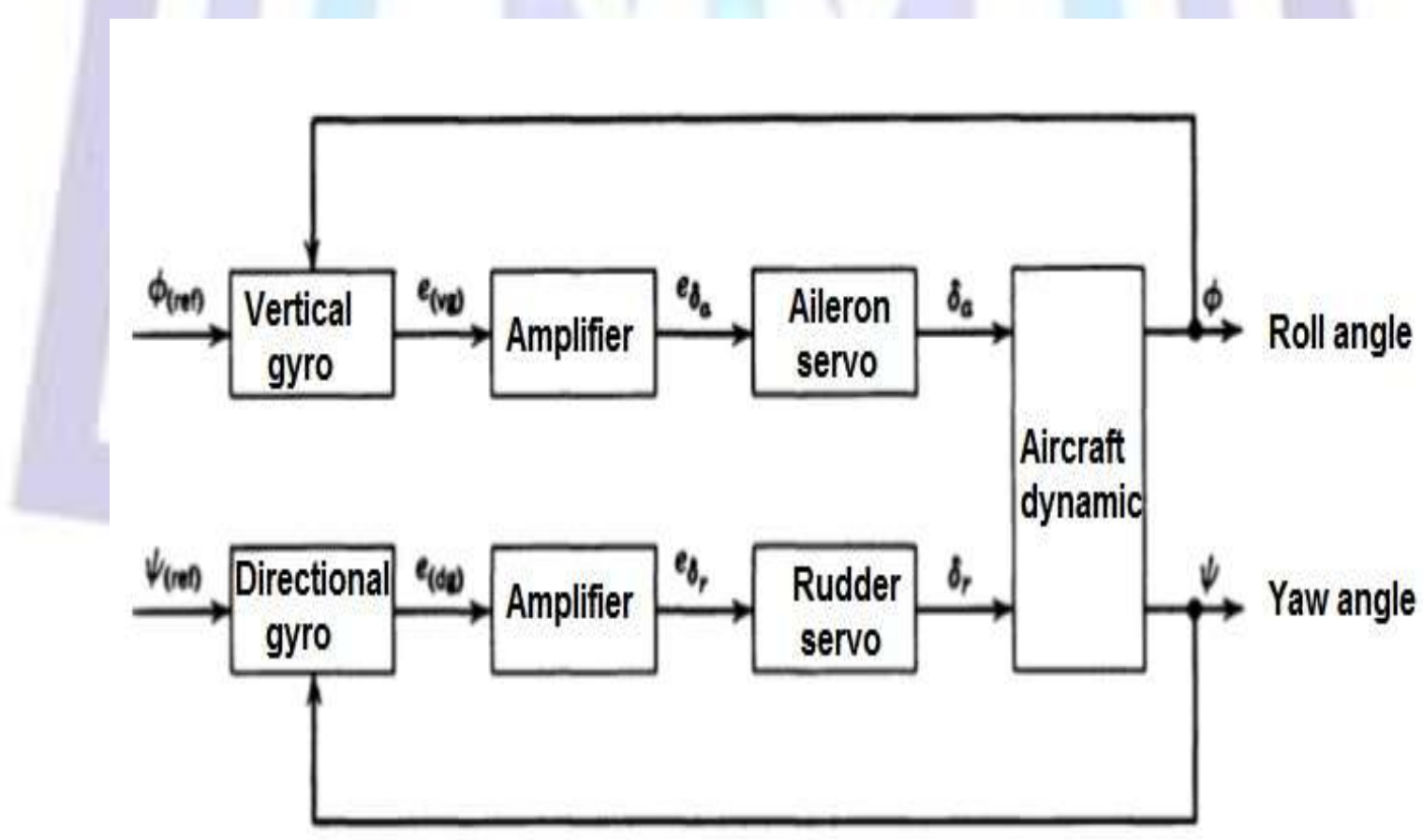

Figure (1): The Lateral mode of Autopilot system

In lateral dynamics of the jet transport aircraft, there are three equations where the first two are moment equations in $L$ and $\mathrm{N}$ directions, while $\mathrm{Y}$ is the force equation in $\mathrm{OY}$ direction

$$
\begin{aligned}
& \Sigma \Delta f_{y=} m\left(v^{\prime}+U R-W P\right) \\
& \Sigma \Delta L^{\prime}=P^{\prime} I_{X}-R^{\prime} J_{X Z}+Q R\left(I_{z}-I_{Y}\right)-P Q J_{X Z} \\
& \Sigma \Delta N=R^{\prime} I_{Z}-P^{\prime} J_{X Z}+P Q\left(I_{Y}-I_{X}\right)+Q R J_{X Z}
\end{aligned}
$$


In lateral dynamics of the jet transport aircraft, linearized and Laplace transformed equation of motion has been used for analysis as given in Equation (1) [5]

$\left.L=\left(\left(I_{x} s q b\right) S^{2}-(b / 2 u) C_{l p} s\right) \Phi^{\prime}(s)+\left(\left(-I_{x z} s q b\right) S^{2}\right)-(b / 2 u) C_{\mid r} s\right) \psi^{\prime}(s)-C_{1 B} \beta^{\prime}(s)=0$

$\mathrm{N}=\left(\left(-I_{x z} / \mathrm{sqb}\right) S^{2}+(\mathrm{b} / 2 \mathrm{u}) \mathrm{C}_{n p} \mathrm{~s}\right) \Phi^{\prime}(\mathrm{s})+\left(\left(\mathrm{I}_{\mathrm{z} / \mathrm{sqb}} \mathrm{S}^{2}\right)-(\mathrm{b} / 2 \mathrm{u}) \mathrm{C}_{\mathrm{nr}} \mathrm{s}\right) \Psi^{\prime}(\mathrm{s})-\mathrm{C}_{\mathrm{n} B} \beta^{\prime}(\mathrm{s})=0$

$\left.Y=-C_{y} \Phi \Phi^{\prime}(s)+((m u / s q) S)-C_{y \psi}\right) \psi^{\prime}(s)+\left((m u / s q) S-C_{y \beta}\right) \beta^{\prime}(s)=0$

Where

$\Phi^{`}=$ Change of roll angle

$\Psi^{r}=$ Change of yaw angle

$\beta^{`}=$ Change of sideslip angle

The characteristic properties of jet transport aircraft and stability derivatives are given in Table (1) and (2), respectively [5].

Table (1): Characteristic properties of jet transport aircraft in lateral mode

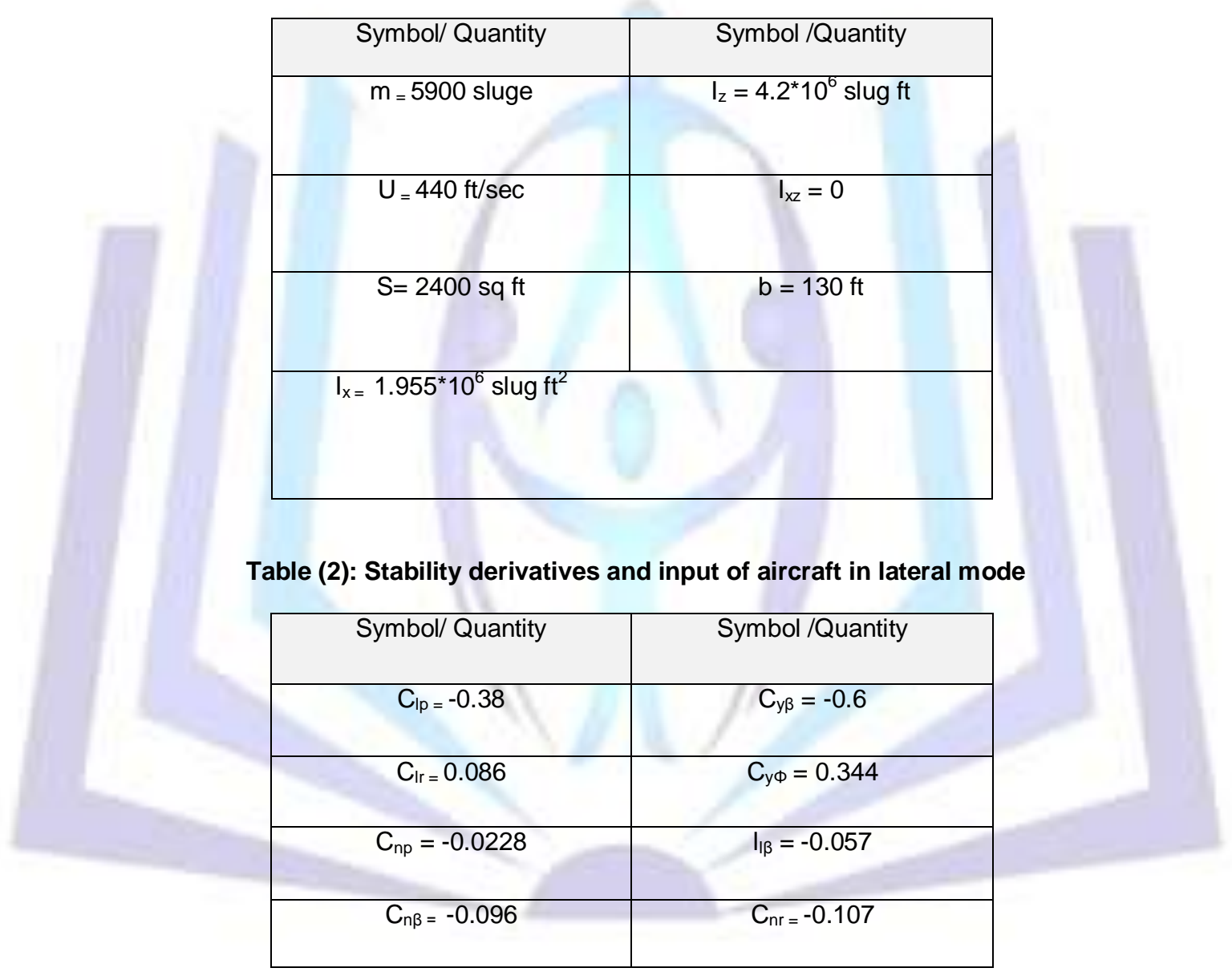

Eq. (2) becomes:

$\left(0.0272 s^{2}+0.055 s\right) \Phi(s)-0.0128 s \Psi(s)+0.057 \beta(s)=0$

$0.0033 s \Phi(s)+\left(0.058 s^{2}+0.015 s\right) \Psi(s)-0.096 \beta(s)=0$

$-0.34 s \Phi(s)+4.7 s \Psi(s)+(4.7 s+0.6) \beta(s)=0$

Arrange in Equations (3) on the matrix to calculate the determinant of the matrix to get:

$\left|\begin{array}{lll}0.0272 s^{2}+0.055 s & -0.0128 s & 0.057 \\ 0.0033 s & 0.058 s^{2}+0.015 s & -0.96 \\ -0.34 s & 4.7 s & 4.7 s+0.6\end{array}\right|=0$


Expanding this determinant the following quadratic equation is obtained

$S^{5}+2.44 S^{4}+2.51 S^{3}+3.68 S^{2}-0.0152 S=0$

By solving the equation (5) to get three real roots and a pair of complex roots $\mathrm{s}\left(\mathrm{s}^{2}+0.380 \mathrm{~s}+1.813\right)(\mathrm{s}+2.09)(\mathrm{s}-0.004)$

There are three types of possible lateral-directional dynamic motion: roll subsidence mode, spiral mode, and Dutch roll mode. The first real root in equation (6) describes the non oscillatory spiral mode used to find the roll angle, the second real root describes the non-oscillatory roll subsidence mode used to find the roll rate and the pair of complex roots describes the oscillatory Dutch roll mode used to find the yaw angle. The Dutch roll that is observed in the yaw rate and sideslip response from an aileron deflection is caused by the yawing moment resulting from the aileron deflection.

\section{4- DISTURBANCE ON AUTOPILOT}

The study of flight is usually divided into two groups: disturbance and space flight system. The two have developed separately over the last period. Atmospheric disturbances undesirably influence aircraft motion and flying safety. The estimate of getting the aircraft response and the equivalent loads in gusts and turbulence is not novel [6]. In that situation were some possible challenges in the previous to use active control technology to counteract atmospheric disturbances [7]. In this paper, disturbances are grouped into three payloads which facing the aircraft during the flight. The first payload is failure in sensor, the second payload is failure in controller and the third payload is disturbances. These payloads used as input to fuzzy set system and the stability of the autopilot used as output to fuzzy set system. Studying the system after the addition of the atmospheric disturbances in lateral dynamic of autopilot have been done by using a white noise.

\section{5- FUZZY SET}

Fuzzy sets are sets whose essentials have degrees of membership. Fuzzy sets were introduced simultaneously by Lotfi A. Zadeh and Dieter Klaua in 1965 as an extension of the classical notion of set [8]. A fuzzy set is an ordered set which connects each value of a variable to its grade of membership in the set. The grade of membership is represented by the membership function. These memberships have several shapes such as triangular or trapezoidal shaped.

Figure (2) shows the system architecture created using the fuzzy set. Three main steps are involved in designing a system architecture using the fuzzy logic [9].

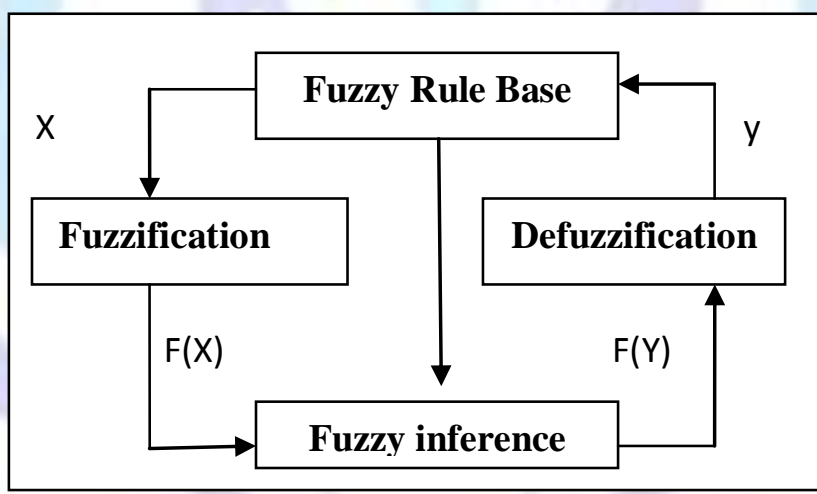

Figure (2): System architecture created using the Fuzzy Set method

(1)Fuzzification: fuzzification involves two processes: derive the membership functions for input and output variables and represent them with linguistic variables. The fuzzification section that receives the input data $x$ and transforms them into $\mathrm{F}(\mathrm{X})$ by the use of different membership functions; F could be linear, Gaussian, etc. Fuzzification allows the grouping of large data sets per membership, and the range of all of the membership functions must describe all of the data sets.

(2) fuzzy inference: The conclusion or control output derived from the combination of input, output membership functions and fuzzy rules is still a vague or fuzzy element, and this process in called fuzzy inference The fuzzy rule is represented by a sequence of the form IF-THEN, the input $F(X)$ is connected to the output $F(Y)$ by a series of rules.

(3)Defuzzification: The defuzzification process is meant to convert the fuzzy output back to the crisp or classical output to the control objective. The output $\mathrm{F}(\mathrm{Y})$ is obtained in fuzzy space from the inference block, and the desired output $y$ is obtained from the defuzzification block. The set up on Fuzzy Set in MATLAB is illustrated in four steps to create a fuzzy controlled machine:

Step one: In this step choose three inputs (controllers, sensors, and disturbances) and one output (stability of autopilot) as shown in Figure (3) 


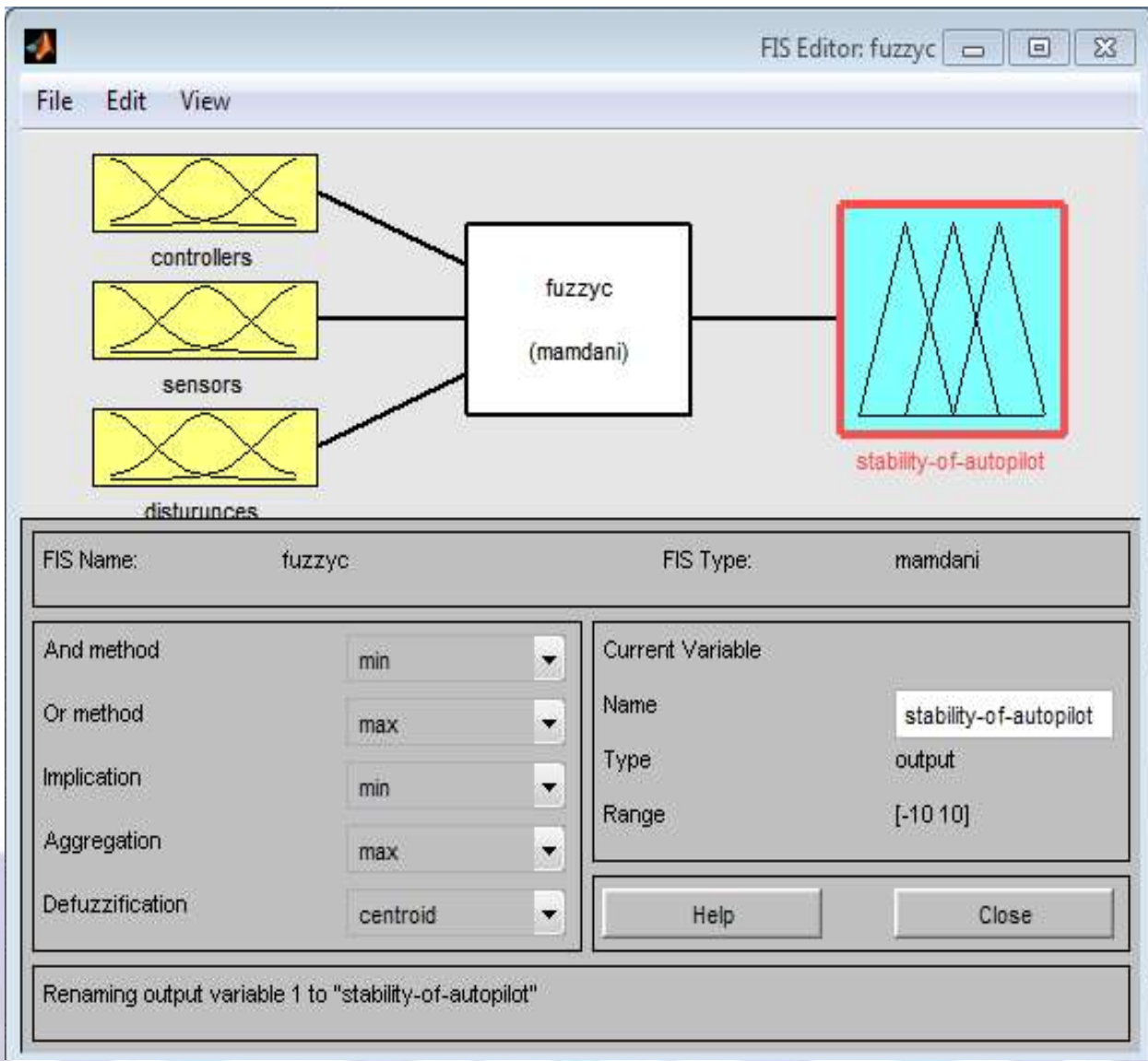

Figure (3): Applied Inputs to the controller

Step two: Determine the membership function of each input (Low, Medium, and High) as illustrated in Figure (4)

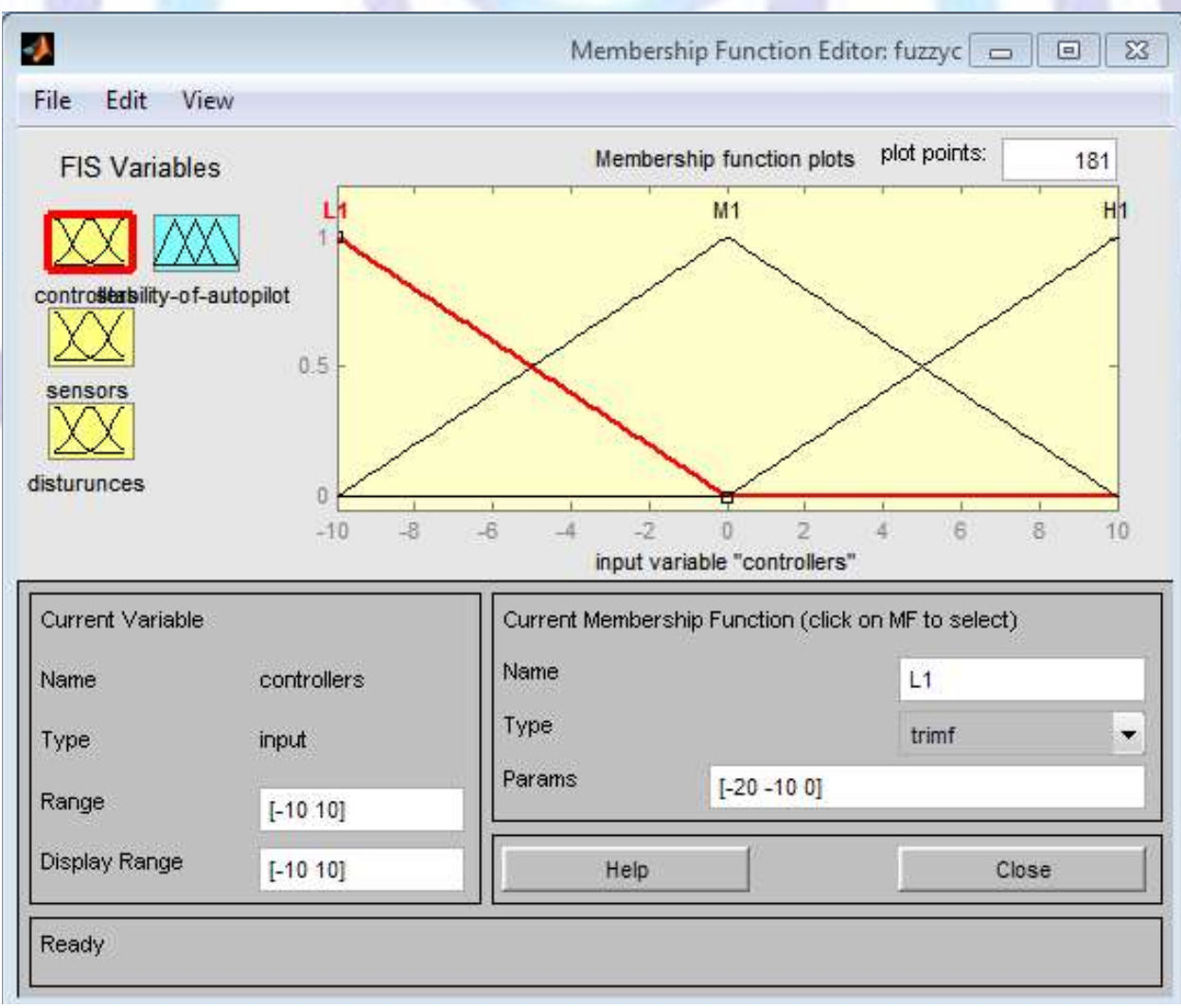

Figure (4): Membership function of the three inputs to the fuzzy logic controller 
Step three: : Edit the list of rules that defines the behavior of the system when fuzzy system have three inputs each input has three member ship (low ,medium, high ) then there are 27 rules as shown in Figure (5)

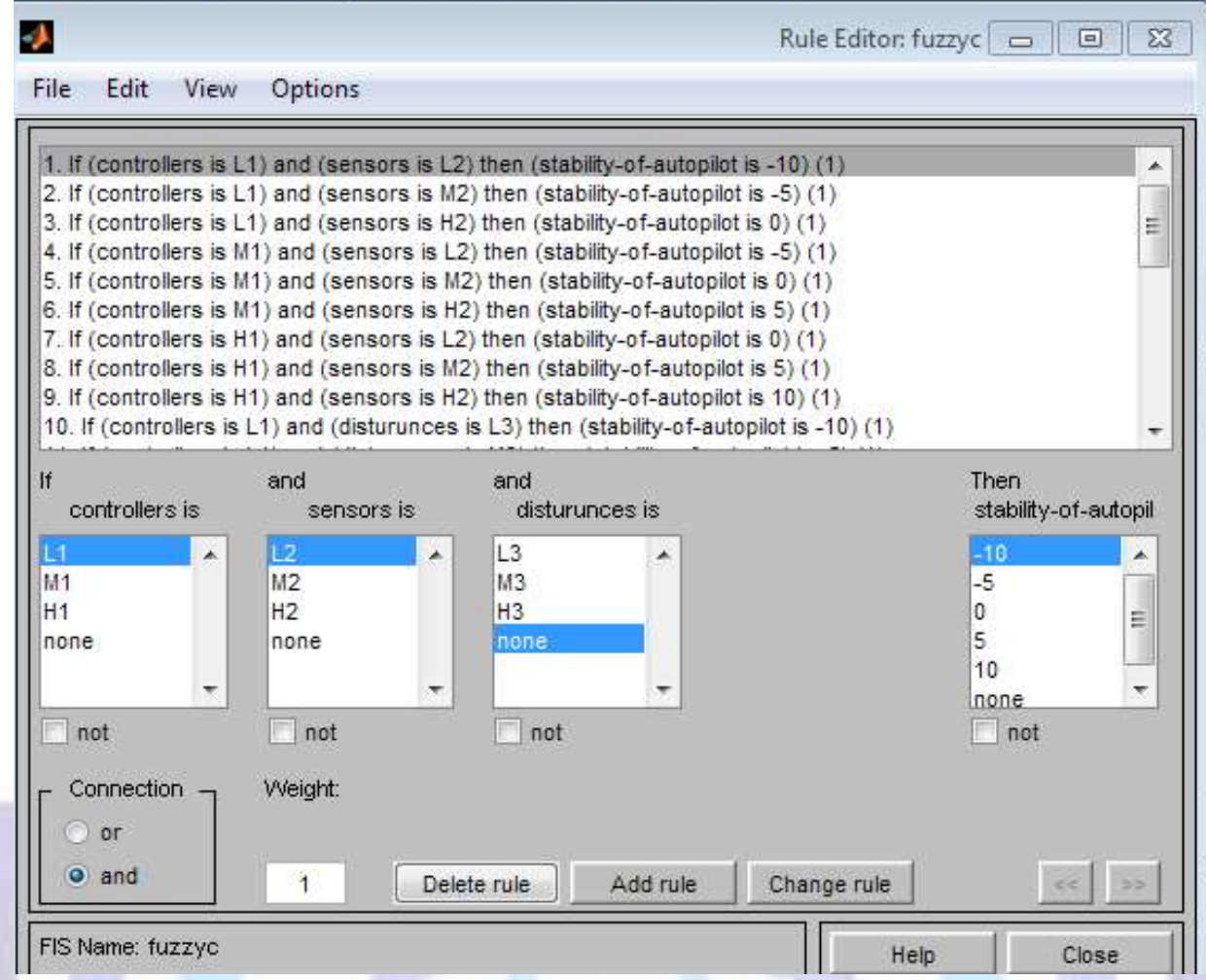

Figure (5): Rules of Fuzzy logic controller

Step four: View the fuzzy inference diagram as shown in Figure (6)

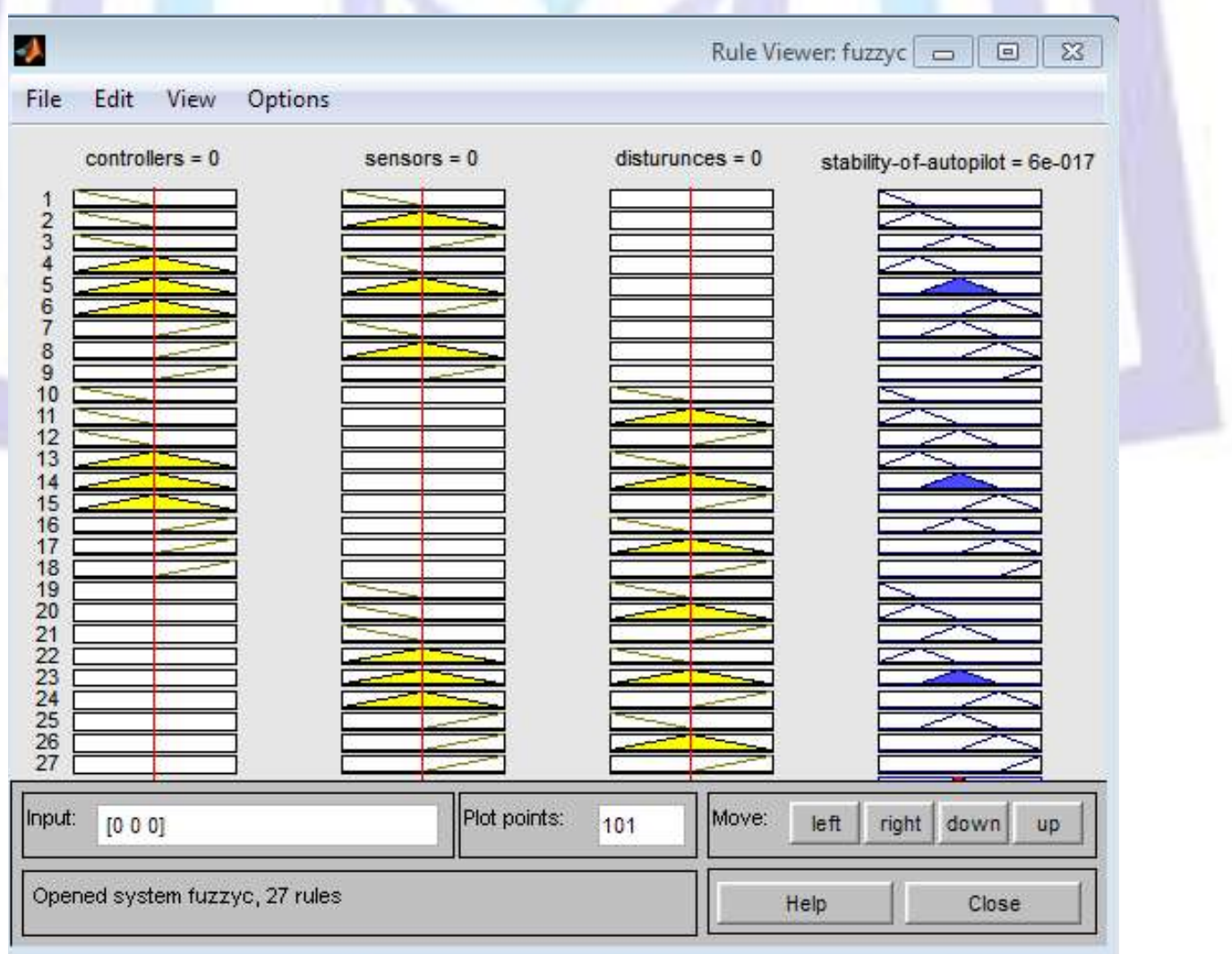

Figure (6): Rule viewer for the fuzzy logic controller 


\section{6-SIMULATION AND RESULTS}

The total simulation of roll angle with case of stability, case of disturbances and case of fuzzy set controller, is illustrated in Figures (7), (8), (9) and (10).

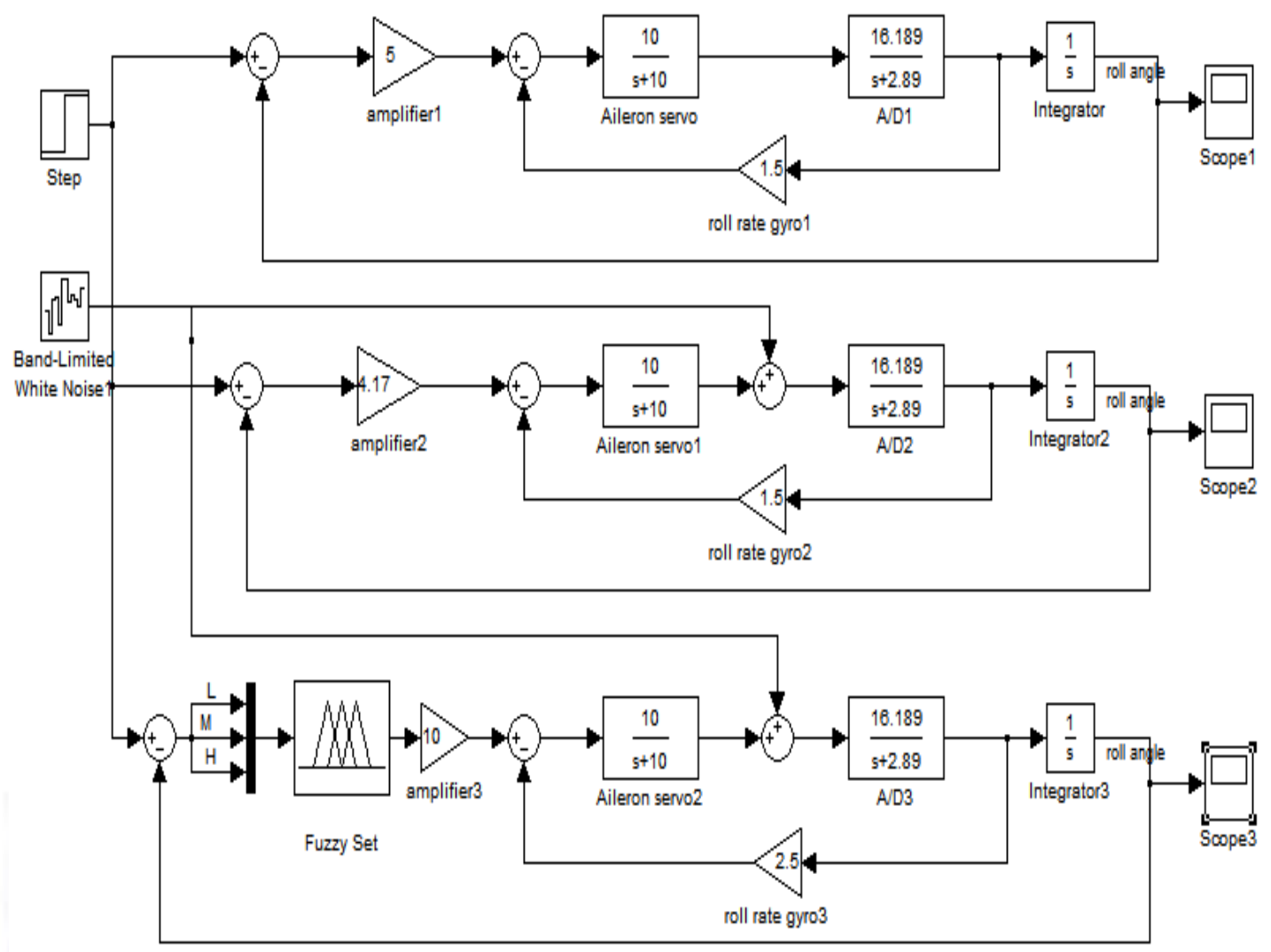

Figure (7): The total simulation of roll angle in lateral dynamic

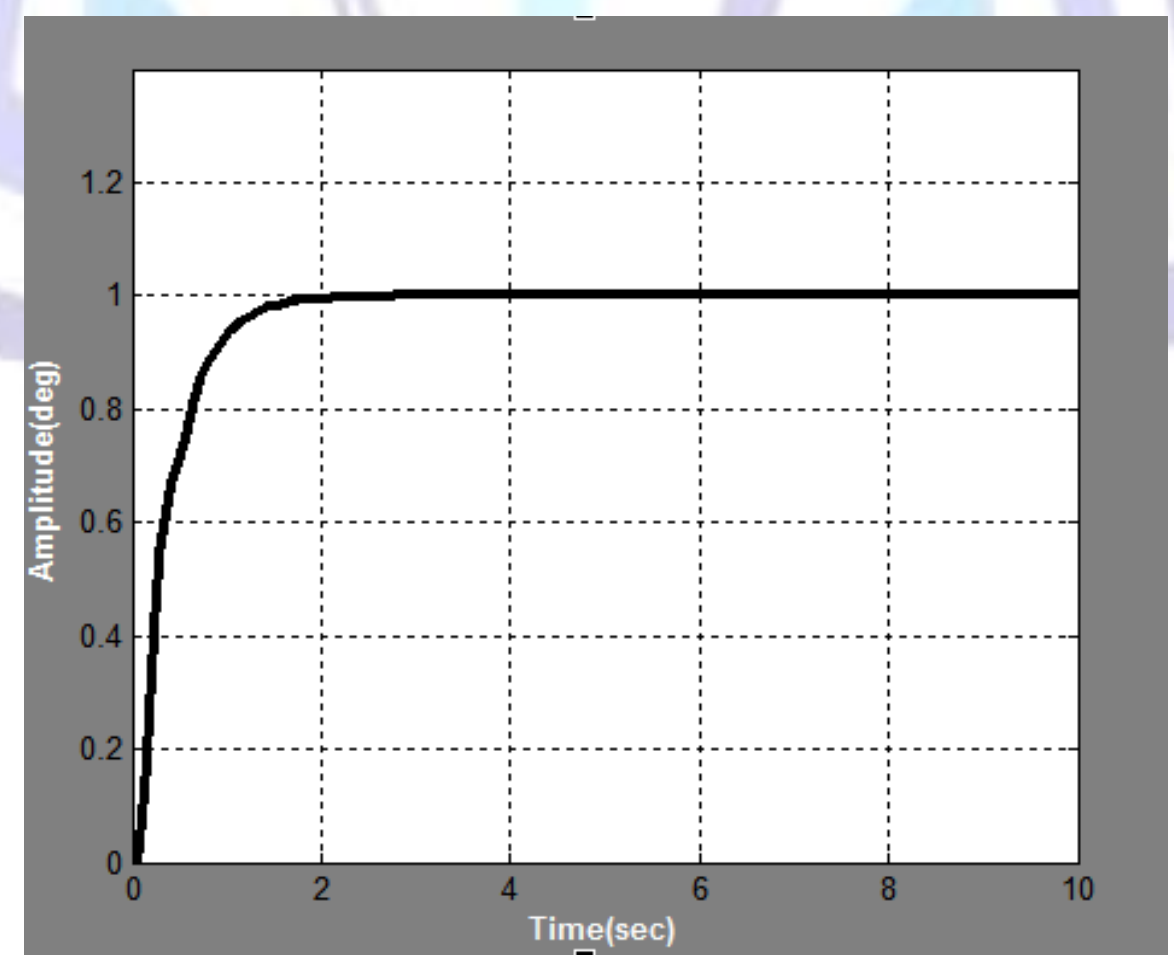

Figure (8): The response of roll angle in stability case 


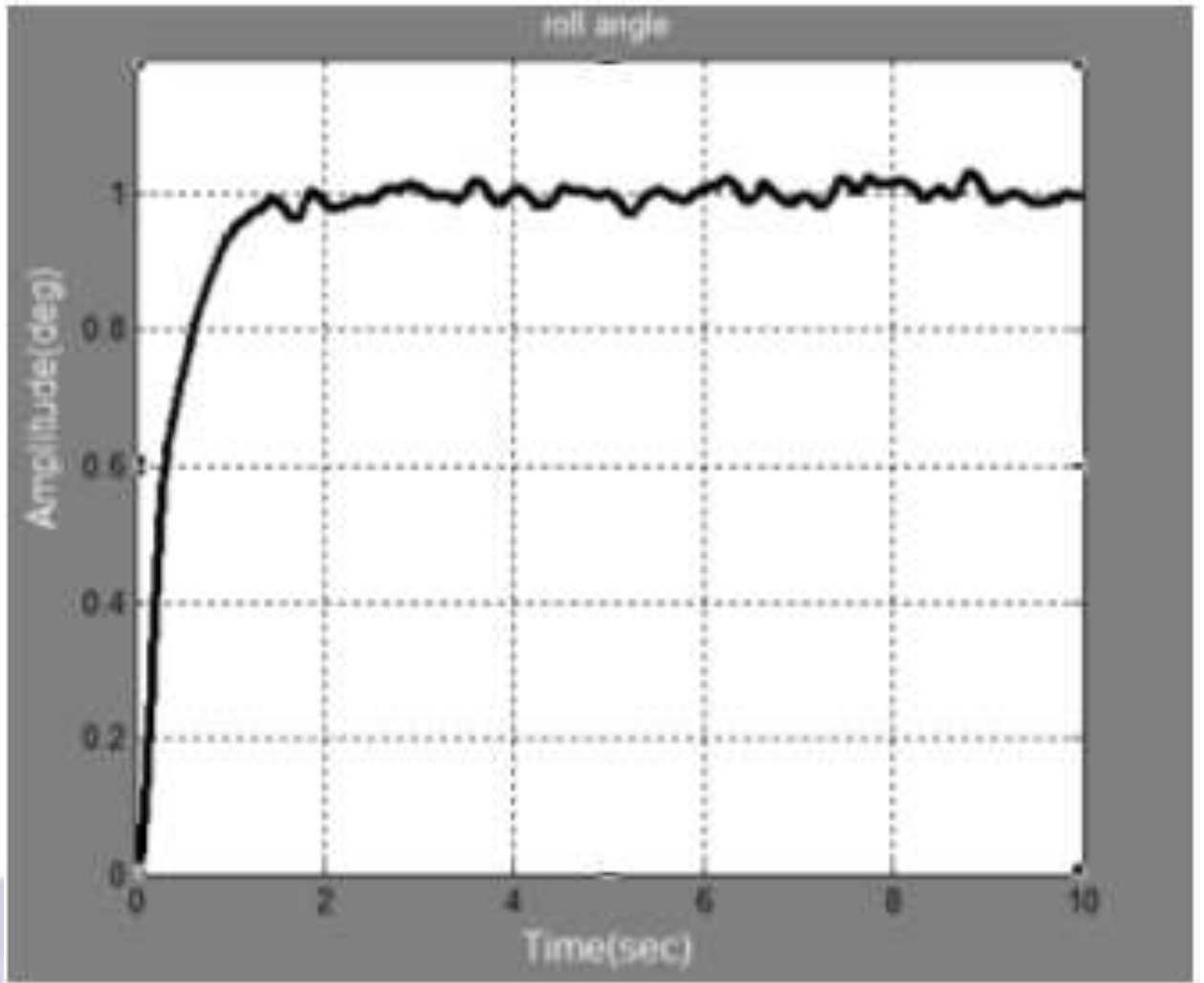

Figure (9): The response of roll angle in disturbance case

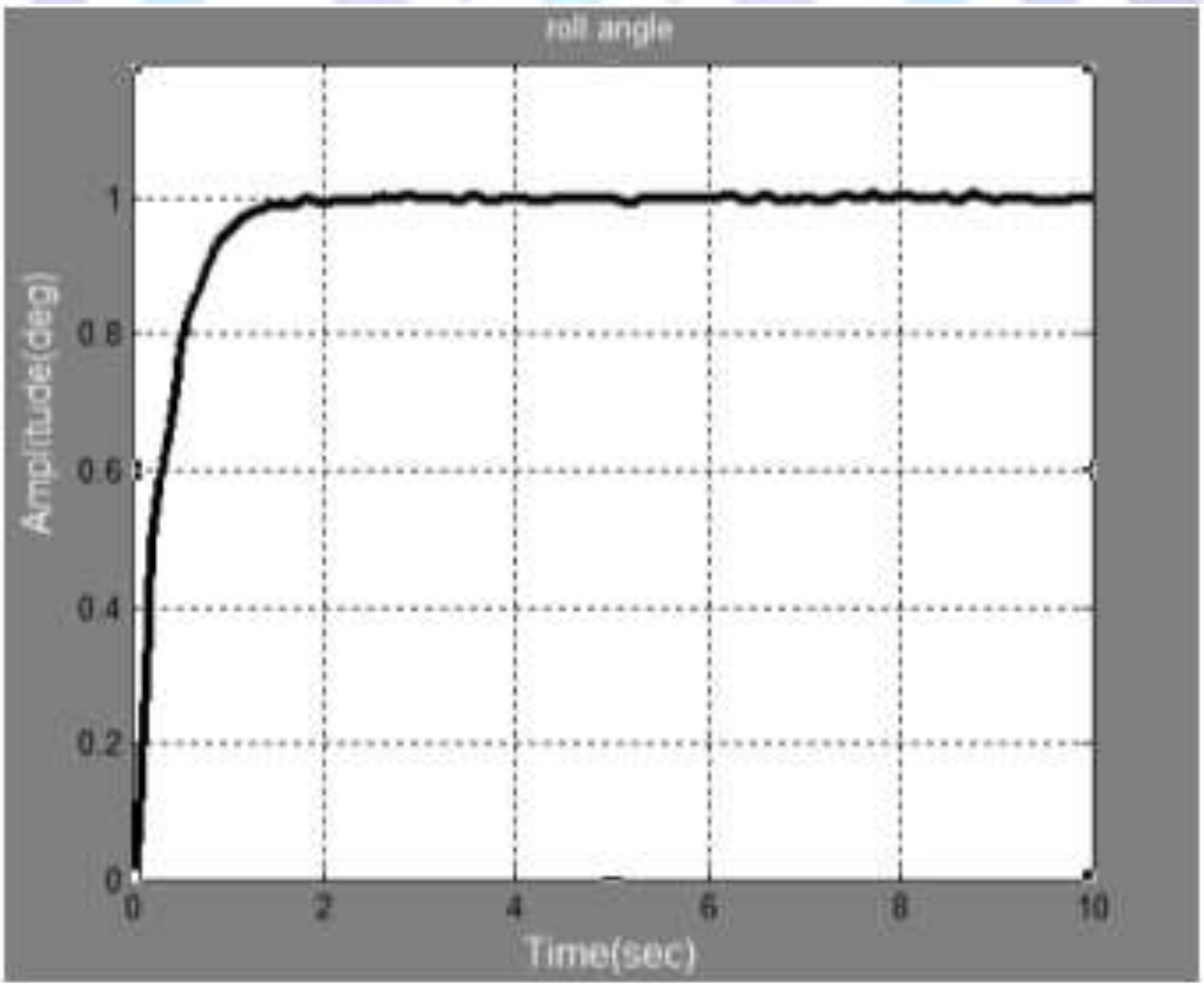

Figure (10): The response of roll angle in fuzzy set controller case

The performance index of roll angle in all previous cases as shows in tables (3) 
Table (3): Summary of performance characteristic of roll angle

\begin{tabular}{|c|c|c|c|}
\hline \multirow{2}{*}{$\begin{array}{c}\text { Performance } \\
\text { Index }\end{array}$} & \multicolumn{3}{|c|}{ Roll angle } \\
\cline { 2 - 4 } & Stable & Disturbances & $\begin{array}{c}\text { Fuzzy set } \\
\text { Controller }\end{array}$ \\
\hline $\begin{array}{c}\text { Delay time, } \\
\mathrm{t}_{\mathrm{d}}(\mathrm{sec})\end{array}$ & 0.2375 & 0.263 & 0.2296 \\
\hline $\begin{array}{c}\text { Rising time, } \\
\mathrm{t}_{\mathrm{r}}(\mathrm{sec})\end{array}$ & 1.2 & 1.285 & 1.383 \\
\hline $\begin{array}{c}\text { Setting time, } \\
\mathrm{t}_{\mathrm{s}}(\mathrm{sec})\end{array}$ & 1.63 & Unstable & 1.72 \\
\hline $\begin{array}{c}\text { Peak time, } \\
\mathrm{t}_{\mathrm{p}}(\mathrm{sec})\end{array}$ & $1 \mathrm{~s}$ & 1.43 & 1.508 \\
\hline $\begin{array}{c}\text { Maximum } \\
\text { overshoot } \mathrm{m}_{\mathrm{p}}\end{array}$ & - & - & - \\
\hline $\begin{array}{c}\text { Steady state } \\
\text { error, ess }\end{array}$ & 0 & 0.0037 & 0.0017 \\
\hline
\end{tabular}

The total simulation of yaw angle with case of stability, case of disturbances and case of fuzzy set (lateral controller is illustrated in Figures (11), (12), (13) and (14).

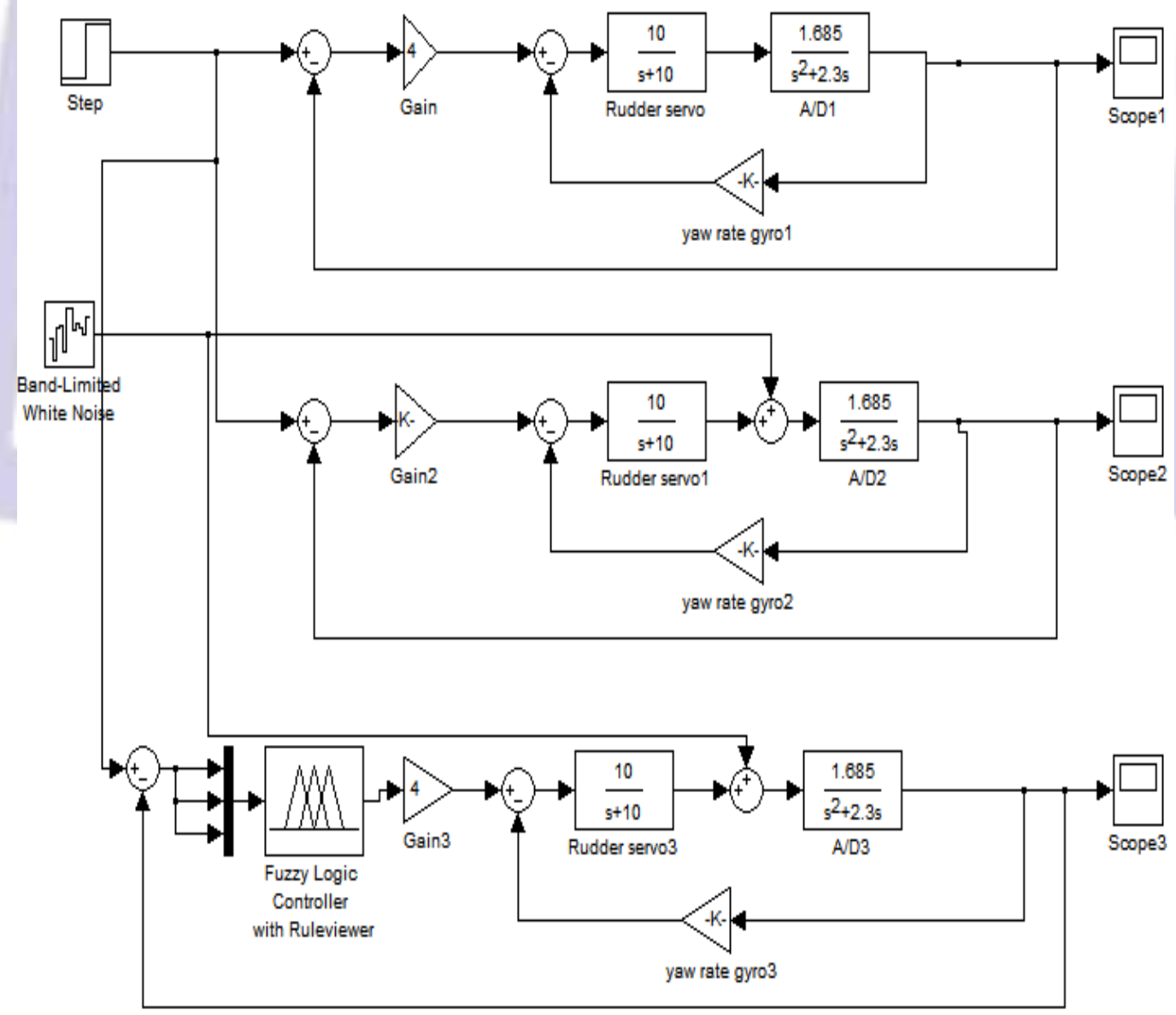

Figure (11): The total simulation of yaw angle in lateral dynamic 


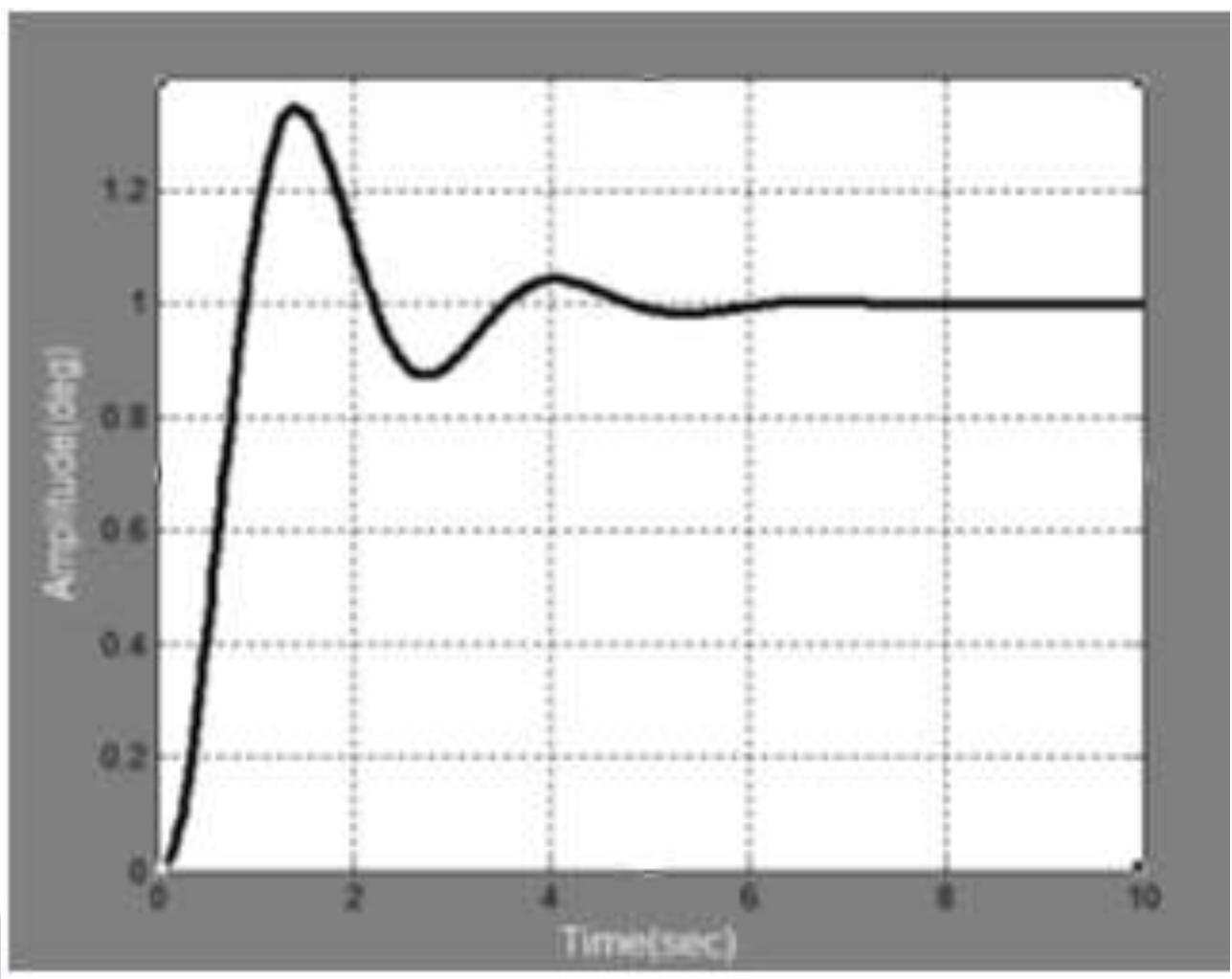

Figure (12): The response of yaw angle in stability case

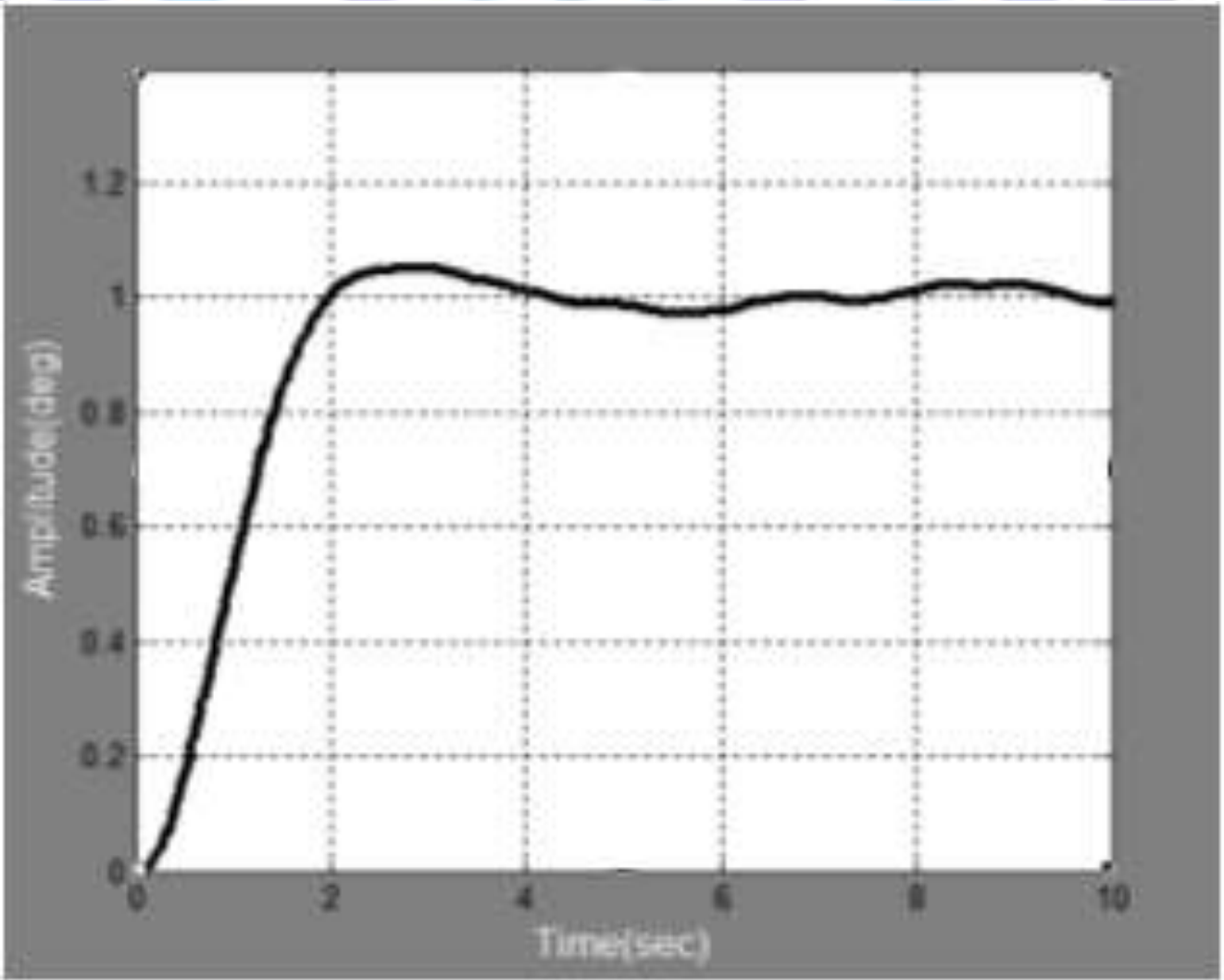

Figure (13): The response of yaw angle in disturbance case 


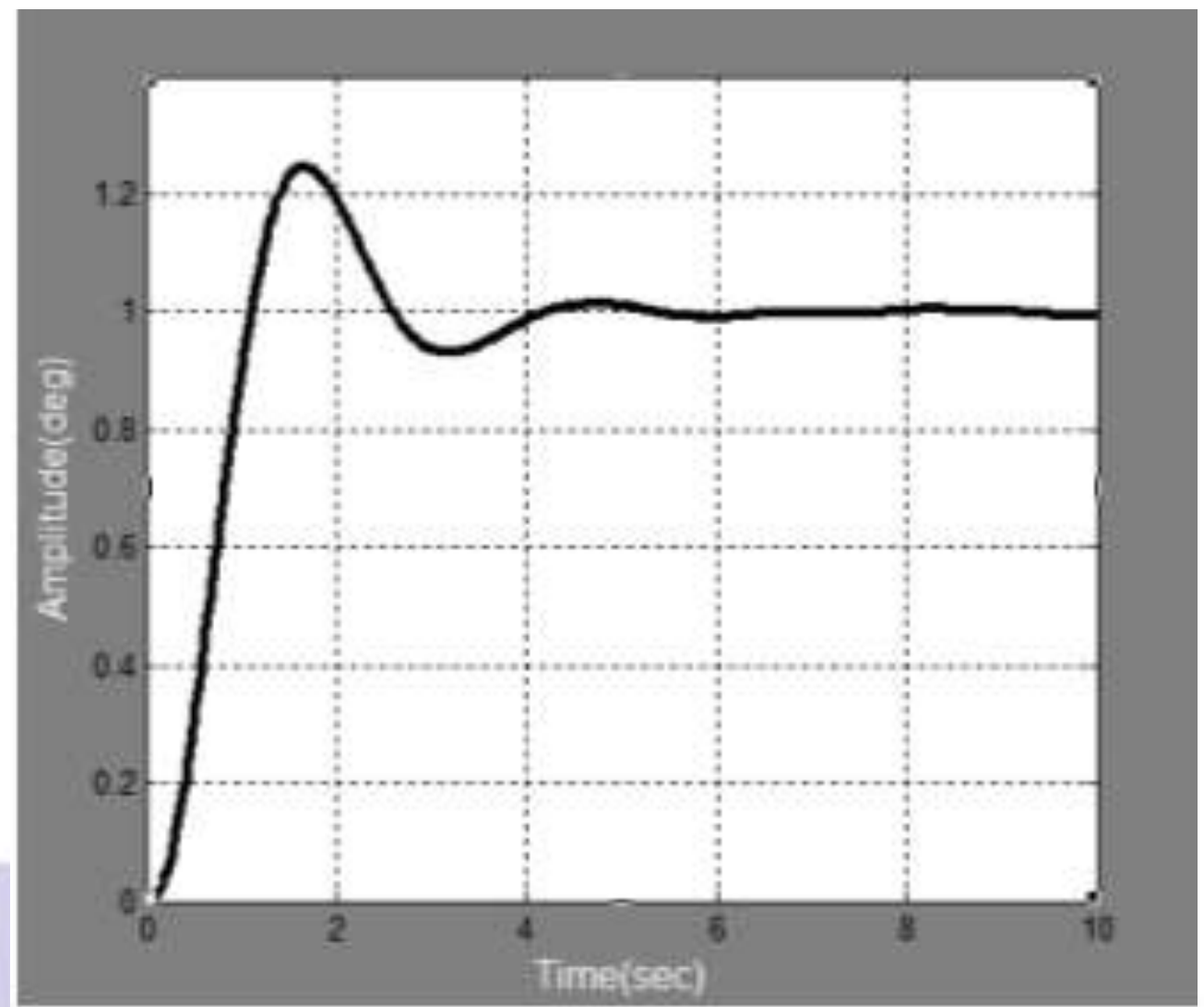

Figure (14): The response of yaw angle in fuzzy set controller case

The performance index of yaw angle in all previous cases as shows in tables (4)

Table (4): Summary of performance characteristic of yaw angle

\begin{tabular}{|c|c|c|c|}
\hline \multirow{2}{*}{$\begin{array}{l}\text { Performance } \\
\text { Index }\end{array}$} & \multicolumn{3}{|c|}{ Yaw angle } \\
\hline & Stable & $\begin{array}{l}\text { Atmospheric } \\
\text { Disturbances }\end{array}$ & $\begin{array}{l}\text { Fuzzy set } \\
\text { Controller }\end{array}$ \\
\hline $\begin{array}{l}\text { Delay time, } \\
t_{d}(\mathrm{sec})\end{array}$ & 0.566 & 0.9728 & 0.6708 \\
\hline $\begin{array}{l}\text { Rising time, } \\
\mathrm{t}_{\mathrm{r}}(\mathrm{sec})\end{array}$ & 0.9 & & 1.1 \\
\hline $\begin{array}{l}\text { Setting time, } \\
t_{s}(\mathrm{sec})\end{array}$ & 5 & 6.6 & 5.151 \\
\hline Peak time, $t_{p}(\mathrm{sec})$ & 1.433 & 2.768 & 1.673 \\
\hline $\begin{array}{c}\text { Maximum } \\
\text { overshoot } m_{p}\end{array}$ & 1.345 & 1.06 & 1.247 \\
\hline $\begin{array}{l}\text { Steady state } \\
\text { error, ess }\end{array}$ & 0.0023 & 0.0121 & 0.0088 \\
\hline
\end{tabular}




\section{7-CONCLUSION}

In this paper, we used fuzzy set as a controller in lateral dynamic (roll angle and yaw angle) of autopilot system to control the impact of disturbances which possibly force the pilot during the flight. The response of roll and yaw angles after the effect of disturbance on the system became unstable but the fuzzy set case gives small delay time, rising time, settling time, and steady state error that goes to zero. Fuzzy set controller is considerably good and has a good disturbance rejection.

\section{REFERENCES}

[1] Michael V. Cool. 2007.Flight Dynamic Principle. $2^{\text {nd }}$, Published by Elsevier Ltd.

[2] Zhongkui Sun, Zhang Hao, Chen Lei and Wang Lili. 2012.Creating Time-Varying Fuzzy Control Rules Based on Data Mining. Maxwell Scientific Organization.

[3] Rauw, Marc. 2001. A Simulink Toolbox for Flight Dynamics and Control Analysis. Marc Rauw.

[4] David F. Anderson and Scott Eberhardt.2001. Understanding Flight. New York. McGraw-Hill.

[5] John H. Blake lock.1991. Automatic Control of Aircraft and Missiles. $2^{\text {nd }}$ edition, A Wiley Interscience publication.

[6] Uma in Ahsun.1996. Dynamic characterization and Active Control of Unstarts in a Near-Isentropic Supersonic Intet .Lahore Pakistan .

[7] Ashish Tewari .2007. Atmospheric and Space Flight Dynamics Modeling and Simulation with meth lab and Simulink. Birkhauser Boston.

[8] Shivangi Sengar, Sakshipriya, Urvashi Khatuja.2012. Fuzzy Set Approaches to Data Mining of Association Rule. International Journal of Computer Science and Informatics.

[9] K. David Solomon Raj, Goutham Tattikota.2013.Design of Fuzzy Logic Controller for Auto landing Application. International Journal of Scientific and Research Publications. 